\section{PWE-052 WHY COLONOSCOPY DOES NOT PREVENT RIGHT SIDED COLORECTAL CANCER?}

doi:10.1136/gutjnl-2013-304907.341

1."L Krupa, ${ }^{1} \mathrm{R}$ Tighe, ${ }^{2} \mathrm{G}$ Phillips. ' Gastroenterology, Norfolk and Norwich University Hospital, Norwich; ${ }^{2} E S O A$, East of UK Screening OARC, Cambridge, UK

Introduction Colonoscopy is widely used for colorectal cancer screening and prevention. There is good evidence that it is associated with lower CRC mortality due to fewer deaths from left-sided cancers. Unfortunately it seems to be less effective in preventing right-sided colorectal cancers (1.2). There are several plausible causes for this finding. One of the reasons could be that right sided lesions are more difficult to detect due to different morphological characteristics.

Methods The adenoma detection rates (ADR) across the East of England within the Bowel cancer screening programme are monitored as part of the OA process. 30 colonoscopists across 8 screening centres acheived ADRs for 2012 varying from $60.1 \%$ to $32.7 \%$ with $26 / 30$ above the OA standard of $35 \%$ ADR. We looked in detail at the ADRs of two colonoscopists in Norwich, who acheived ADRs of $60.1 \%$ (endoscopist A) and $35.3 \%$ (endoscopist B), over a 2 year timespan.

Results Endoscopist A performed 441 colonoscopies and endoscopist B 544 .

Endoscopist A detected 815 adenomas and endoscopist B 545 $(p<0.0001)$, with endoscopist A recorded no adenoma or cancer in $125 / 441$ patients vs endoscopist B 251/544 ( $p<0.0001$ ). Each endoscopist detected similar numbers of pedunculated adenomas (112/441 vs $130 / 544)(p=0.5866)$ but endoscopist A identified significantly more sessile adenomas 700/441 vs 409/544 ( $p<0.0001$ ).

Adenomatous polyps were graded by size : $>10 \mathrm{~mm} \mathrm{~A} \mathrm{113/441} \mathrm{vs}$ B 95/544 ( $p=0.0018) ; 6-10$ mm A 165/441 vs B 132/544 ( $<<0.0001)$; and $<6 \mathrm{~mm} \mathrm{A537/441} \mathrm{vs} \mathrm{B} \mathrm{318/544} \mathrm{(}<<0.0001)$.

Endoscopist A detected more adenomas proximal to the splenic flexure 425/441 vs B 205/544 ( $p<0.0001$ ), whereas the ADRs distal to the splenic flexure were similar A330/441 vs B $340 / 544$ $(\mathrm{p}<0.0001)$

Endoscopist A had a higher completion rate of $99.7 \%$ compared with $94.67 \%$ for endoscopist B $(p<0.0001)$. Withdrawal times were similar (for procedures in which no polyps were found) A $10.59 \mathrm{~min}$ vs B $9.28 \mathrm{~min}$.

Conclusion Sessile polyps in the right colon are commonly overlooked even by expert bcss accredited colonoscopists. Over half the patients discharged from the programme by endoscopist B with a "normal" colon have had a small right sided adenoma overlooked and it seems likely this is the reason that colonoscopy fails to prevent the development of right sided colonic cancer. The current $\mathrm{OA}$ standard for ADR in bcss is too low at $35 \%$. The current JAG OA standard for ADR among the wider colonoscopy community is $10 \%$ and it is likely that this problem is widespread.

Disclosure of Interest None Declared.

\section{REFERENCES}

1. Baxter NN, Goldwasser MA, Paszat LF, et al. Association of colonoscopy and death from colorectal cancer: a population-based, casecontrol study. Ann Intern Med 2009; 150:1-8.

2. Brenner $\mathrm{H}$, Hoffmeister $\mathrm{M}$, Arndt $\mathrm{V}$, et al. Protection from right- and left-sided colorectal neoplasms after colonoscopy: population-based study. J Natl Cancer Inst 2010; 102:89-95.

\section{PWE-053 EUS-FNA AND PET-CT IN THE STAGING OF MEDIASTINAL LYMPHADENOPATHY IN MALIGNANT DISEASE}

doi:10.1136/gutjnl-2013-304907.342

1."L A Smith, ${ }^{2} S$ Paterson, 'N Jamieson, ${ }^{3}$ S Ballantyne, 'A J Stanley. 'Gastroenterology, Glasgow Royal Infimary, Glasgow; 'astroenterology, Forth Valley Royal Hospital, Larbert; ${ }^{3}$ Radiology, Gartnaval General Hospital, Glasgow, UK
Introduction EUS+/- FNA is commonly used in the nodal staging of upper GI, lung and other malignancies, but the increasing availability of PET-CT has led to a reduction in its use in some centres. However the accuracy of PET-CT in staging mediastinal nodes in particular is unclear. Our aim was to analyse the concordance of PET-CT and EUS+FNA in the staging of mediastinal nodes in malignant disease in one tertiary referral centre.

Methods Electronic reports for all patients attending for EUS between January 2009 and December 2012 were reviewed. Patients who had both mediastinal node sampling and a PET-CT were included for analysis. Using a positive EUS +FNA result as a definitive diagnosis of lymph node involvement, the accuracy, sensitivity, specificity, positive (PPV) and negative predictive values (NPV) of PET-CT in the staging of mediastinal lymph nodes were calculated. The final staging pathway and patient outcomes were also analysed.

Results A total of 77 nodes in 74 patients were sampled (51M, 23F; median age 66). The primary diagnosis was that of oesophageal cancer in 56 patients (38 adeno and 18 squamous); lung cancer in 11 patients; gastric cancer in 3 patients and other malignancies in 4 patients. The FNA cytology results were positive, negative and indeterminate in $32(42 \%), 39(51 \%)$ and $6(8 \%)$ cases respectively. The cytological indeterminate nodes were excluded from further analysis. The sampled nodes were positive, negative and indeterminate on PET-CT in 49 (64\%), 26 (34\%) and 2 (3\%) cases respectively. For all patients, the sensitivity, specificity, PPV, NPV and accuracy of PET-CT were $97 \%, 54 \%, 63 \%$, 95\% and $73 \%$ respectively. For the subgroup with oesophageal cancer the sensitivity, specificity, PPV, NPV and accuracy were $94 \%, 62 \%, 57 \%, 95 \%$ and $73 \%$. EUS +FNA altered the cancer staging and changed the patient pathway in 14 patients $(20 \%)$.

Conclusion PET-CT has a poor specificity and PPV for the diagnosis of malignant mediastinal lymph nodes, resulting in a high false positive rate and potential over staging. Therefore EUS-FNA remains an essential part of the staging process for these patients

Disclosure of Interest None Declared.

\section{PWE-054 PRELIMINARY EXPERIENCE OF HEMOSPRAY IN THE MANAGEMENT OF DIFFUSE PORTAL HYPERTENSIVE BLEEDING}

doi:10.1136/gutjnl-2013-304907.343

1."L A Smith, 'J Morris, 'A J Stanley. 'Gastroenterology, Glasgow Royal Infimary, Glasgow, UK

Introduction Hemospray is a novel powder licenced in Europe and Canada for endoscopic hemostasis of non-variceal upper gastrointestinal bleeding. Portal hypertensive gastropathy (PHG), enteropathy or colopathy develop in many patients with portal hypertension. These conditions often present with chronic anaemia. However they can also result in acute blood loss which is difficult to treat due to the diffuse nature of bleeding.

Methods We present data from 4 consecutive patients presenting to our institution with acute haemorrhage secondary to non-variceal diffuse portal hypertensive bleeding, which was treated with Hemospray.

Results Patient 1- a 67 year old man with alcoholic liver disease and cirrhosis attended for variceal screening gastroscopy. At the time he was found to have active bleeding from severe PHG. Hemospray was applied to this area achieving hemostasis, with no complications. Elective repeat gastroscopy at 4 weeks showed moderate PHG with no active bleeding and he had no clinical rebleeding by 6 weeks. Patient 2- a 74 year old lady with cryptogenic cirrhosis and transfusion dependent anaemia secondary to PHG despite betablockers, presented with an acute upper gastrointestinal bleed. Gastroscopy showed active bleeding from diffuse antral PHG. Argon beam diathermy failed to achieve hemostasis, therefore Hemospray 